\title{
Pseudo-myotonia and myokymia
}

\author{
R. C. HUGHES AND W. B. MATTHEWS \\ From the Derbyshire Royal Infirmary
}

In 1961 Isaacs described the clinical features and effective symptomatic treatment of a previously unrecognized form of incapacitating muscular rigidity. His title of 'A syndrome of continuous muscle-fibre activity' was not entirely supported by the electrophysiological data, and the condition, while distinctive enough, has no accepted name. No example has been described in Great Britain and the natural history of the disease is little known. We have had the opportunity to observe a patient in whom the clinical course could be traced with some precision for 32 years.

\section{CASE REPORT}

W.W. (DRI 88172) was born in 1914. He was an only child and his parents did not suffer from any form of muscle disease. As a child he suffered from asthma, but was otherwise in good health until at the age of 22 he had an attack of jaundice lasting for six weeks and accompanied by malaise, pale stools, and dark urine.

While recovering from this illness he first noticed stiffness of the fingers and difficulty in relaxing his handgrip. Over the next few months the stiffness spread to involve the muscles of the thighs, the shoulder girdle, the trunk, and face. After walking or running a few yards, movement would become much freer but the stiffness rapidly returned on resting. Coughing was followed by delayed relaxation of the abdominal muscles and he had difficulty in opening the eyes rapidly after forcible closure. There was no dysphagia, difficulty in chewing, or dyspnoea. The muscle spasm was not painful, but he experienced some nocturnal shooting pains in the thighs. About a year after the onset of these symptoms he noticed gradual flexion of the terminal phalanges of both index fingers and the right middle finger.

At the age of 24 he was admitted to the London Hospital under the care of Dr. G. Riddoch. ${ }^{1}$

The only abnormality on general examination was increased sweating which was thought to smell 'acid'. The muscles of the shoulder girdle, the abdominal wall, and the thighs were noted to stand out prominently and the deltoid muscles in particular were thought to be hypertrophied. The intrinsic hand muscles were slightly thin and there was possibly slight distal weakness of all limbs. He had slight bilateral ptosis. There was marked

${ }^{2}$ We are indebted to Dr. C. J. Earl for a copy of the case notes made at this time. resistance to passive movement of the limbs, the abdominal wall was noted to be rigid and his gait was 'spastic'. The quadriceps muscle was in an 'almost constant state of contraction'. 'Fibrillation' was seen in the vastus internus.

Delayed relaxation after contraction was particularly marked in the handgrip but was also present on closure of the eyes. On repeated movement, relaxation became increasingly rapid and fine movements of the fingers became possible. Percussion myotonia could not be elicited. The flexion deformity of the terminal phalanges of which the patient complained was noted. There was no sensory loss. The tendon reflexes were absent and the plantar reflexes normal.

Investigation included a normal cerebrospinal fluid, a serum calcium of $11 \cdot 1 \mathrm{mg} / 100 \mathrm{ml}$., and a normal calcium balance.

Treatment with oral neostigmin was unhelpful and he was discharged with the diagnosis of myotonia acquisita on treatment with quinine.

He first attended the Derbyshire Royal Infirmary in 1955 at the age of 42 . He had taken quinine intermittently in varying dosage but had detected no definite effect on his stiffness, although he thought that the excessive sweating had diminished. He had been able to continue at work as a chair-frame maker, although with considerable difficulty. His symptoms had not changed for many years but were less severe than in the few years following their onset. While anxious to obtain effective treatment, he was unwilling to undergo any investigation that might cause him to lose time from work as his disability was such that employment was precarious. In particular he did not give permission for a muscle biopsy.

Examination confirmed that virtually the entire musculature of the limbs and trunk was in a continuous state of contraction. Constant writhing fasciculation was extremely prominent, particularly in the thighs and arms and the patient was now aware of this phenomenon. Delayed relaxation after voluntary contraction, improving with repeated movement, was particularly obvious in the handgrip. His gait was slow and stiff. No percussion myotonia was present. The deformity of the fingers (Fig. 1) was now more extensive but was not due to fixed contracture as it could readily be overcome by passive movement. There was no weakness, no wasting of the sterno-mastoid muscles, no testicular atrophy, and no cataract or frontal baldness. Sweating was not excessive, but he suffered from intermittent eczema of the trunk and limbs.

Mephenesin carbamate $500 \mathrm{mg}$ three times a day had 


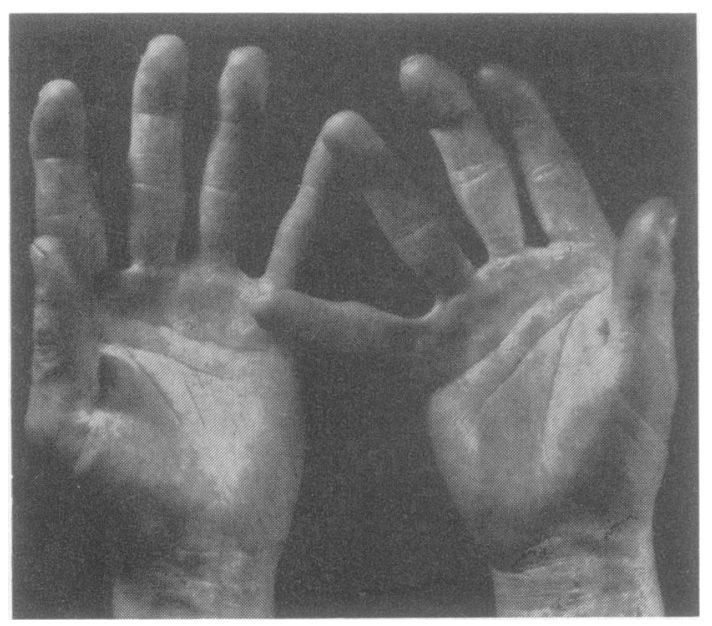

FIG. 1. Photograph of hands showing permanent flexion of terminal phalanges of all fingers on left and of index and middle fingers on right. In addition there is abduction of the left little finger.

no beneficial effect. Procaine amide $600 \mathrm{mg}$ by slow intravenous infusion did not alter the fasciculation but caused some reduction in the rigidity. Oral dosage up to $1 \mathrm{~g}$ four times a day had no convincing effect.

His condition remained unchanged until 1962 when a trial of phenytoin sodium $100 \mathrm{mg}$ four times a day was begun. This produced immediate benefit and when seen in 1967 the patient had found that he could control the stiffness adequately with 200 to $300 \mathrm{mg}$ a day in divided doses, but that if he neglected the treatment the symptoms returned within 24 hours. He had also noticed that any form of alcoholic drink lessened the stiffness and that cold had an adverse effect, although this was not marked. At no time was the stiffness entirely abolished, but there had been a notable improvement in his speed of walking and in his ability to manipulate tools at work.

Examination showed that when taking this dose of phenytoin delayed relaxation of the grip was still detectable. Resistance to passive movement was no longer increased and the muscles were soft with the exception of the forearm muscles which were extremely firm. Fasciculation was still prominent and did not appear to have been influenced by treatment. The deformity of the hands had not changed.

On 17 December 1967 treatment was changed to carbamazepine $100 \mathrm{mg}$ three times a day, rapidly increased to $200 \mathrm{mg}$ three times a day. No toxic effects have occurred in the succeeding six months and the patient is convinced that this form of therapy is superior to phenytoin. He feels that the stiffness is no longer a disability and that if he neglects to take his tablets the stiffness may not return for $\mathbf{4 8}$ hours or longer. Examination, however, fails to show any convincing difference. Fasciculation is still continuous, the forearm muscles are still unduly firm, and delayed relaxation of the grip can still be detected.
INVESTIGATION Investigation was limited by the patient's wish not to be admitted to hospital.

A routine blood count showed: haemoglobin $13 \cdot 5$ $\mathrm{g} / 100 \mathrm{ml}$., WBC 5000/cu.mm, ESR $19 \mathrm{~mm}$ in 1 hours (Westergren), serum creatine phosphokinase $35 \mathrm{mu} . / \mathrm{ml}$ serum (normal 0 to 50 ), serum aldolase $1.8 \mathrm{mu} . / \mathrm{ml}$. (normaf 0 to 6). Serum sodium 138 m-equiv/l., potassium 4.4 م m-equiv/l., chloride $98 \mathrm{~m}$-equiv/l., alkali reserve 24 m-equiv/l., urea $17 \mathrm{mg} / 100 \mathrm{ml}$.

ELECTROMYOGRAPHY EMG using concentric needle electrodes has been carried out on many muscles of the upper limbs at intervals between 1959 and 1968. $\mathrm{No}$ insertion myotonia has ever been observed. Forcefut voluntary contraction was always accompanied by a fulf interference pattern of motor units of normal amplitude and duration. During slight voluntary activity th\& recording sometimes consisted of one or two low voltage discharges repeated at frequencies varying from 40 to 70. a minute, appearing out of proportion to the force exerted (Fig. 2a).

Except in the particular circumstances mentione $\vec{\phi}$ below, activity at rest was always present in the muscles. sampled, both before treatment and on clinically effective doses of phenytoin and carbamazepine. The usual patterif of activity was that at any point sampled two or mores stereotyped discharges could be recorded, firing singlyor in 'multiplets' without any regular frequency (Fig. \$b) Discounting activity apparently originating at sạne distance from the needle point, the amplitude of these discharges exceeded $200 \mu \mathrm{V}$ but was seldom more than $700 \mu \mathrm{V}$. Some discharges were obviously polyphasic $\overline{\text { Wh }}$ of long duration-12 to $15 \mathrm{msec}$ (Fig. 2c).

Voluntary activity was followed by a great increase $\mathrm{i} \hat{\text { p }}$ the density of the spontaneous activity (Fig. 2d an but after approximately 30 seconds was regural succeeded by electrical silence for about one minute? Electrical silence could also be produced by the application of a sphygmomanometer cuff inflated above the systolic blood pressure for seven minutes. On removing the cuff spontaneous activity slowly returned. In the forearm muscles examined activity at rest was nof diminished by effective symptomatic treatment and more surprisingly, the density and duration of the discharge following voluntary activity were also ung changed.

Motor nerve conduction velocity in the right ulna nerve was $67 \mathrm{~m} / \mathrm{sec}$ in the segment from elbow to wrist with a terminal latency of $\mathbf{2 \cdot 3} \mathrm{msec}$. The muscle action potential from abductor digiti minimi was normal.

\section{DISCUSSION}

The clinical features and response to treatment can leave no doubt that our patient suffers from the condition described by Isaacs (1961). The three patients described by Mertens and Zschocke (1968 under the title of 'Neuromyotonie' and the case of: 'pseudo-myotonie' of Sigwald, Raverdy, Fardeats Gremy, Mace de Lepinay, Bouttier, and Danif (1966) unmistakably have the same syndrome. The 


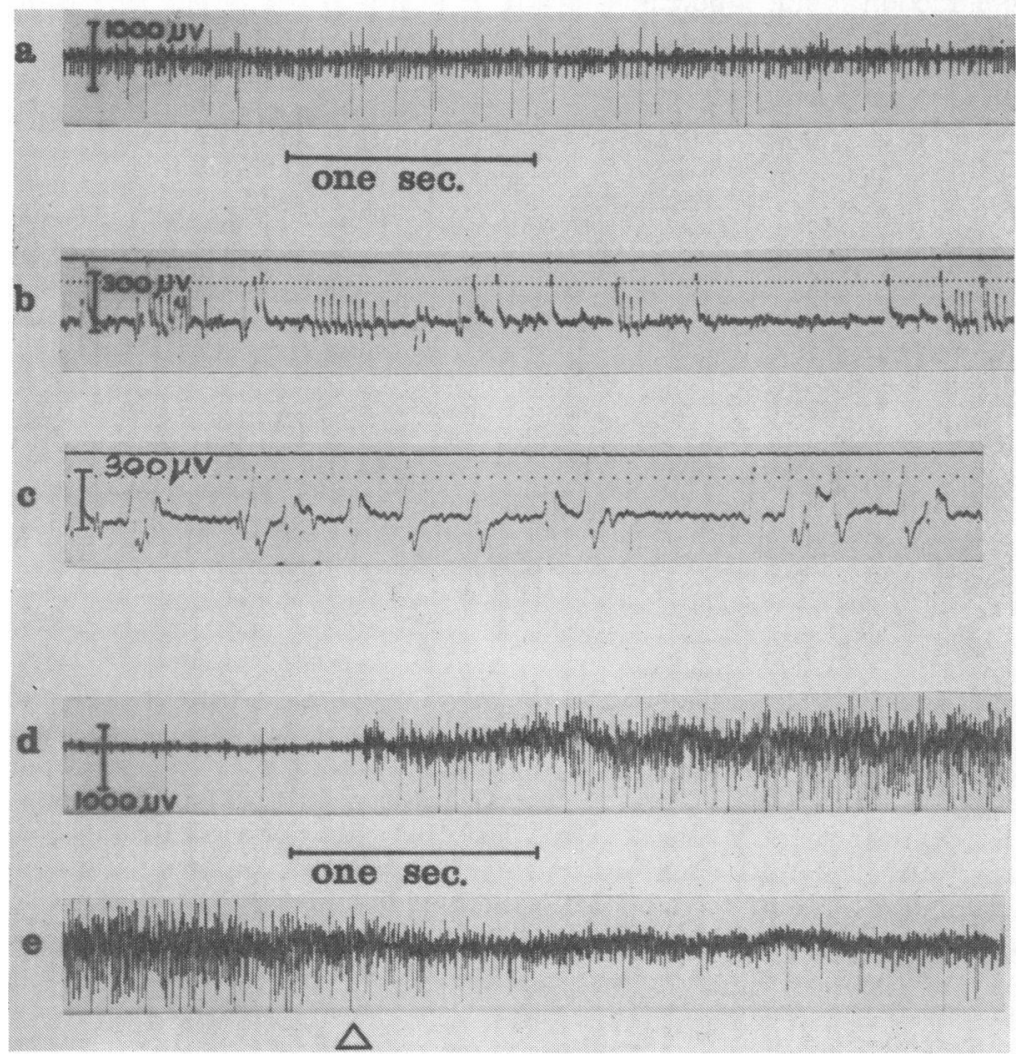

FIG. 2. Electromyogramssampling with concentric needle electrodes.

(a) Extensor digitorum-minimal voluntary contraction. (b) Extensor digitorum - activity at rest consisting mainly of stereotyped monophasic complexes at $300 \mu \mathrm{V}$ amplitude and duration 8-10 $\mathrm{msec}$, but, in addition, bursts of biphasic activity of amplitude $200 \mu \mathrm{V}$ and rate $80-100 / \mathrm{sec}$ (time scale 10 msec intervals). (c) Activity at rest-left abductor digiti minimi showing polyphasic units of amplitude 300-400 $\mu \mathrm{V}$ and duration 15-20 msec (time scale 10 msec intervals). (d) and (e) Extensor digitorum-activity before, during, and immediately after maximum voluntary contraction. Note activity greater than rest activity persists after cessation of active contraction.

In (a), (d), and (e) time scale and amplitude as shown. In (b) and (c) time scale is marked in 10 msec intervals-amplitude as shown.

age of onset has varied from 5 to 53 and both sexes are affected. Usually no precipitating factor can be identified and the onset is gradual. The degree of disability varies, our patient being comparatively mildly affected, while the young woman described by Sigwald et al. (1966) was severely incapacitated and had serious difficulty in swallowing and breathing.

It is unlikely that the full clinical syndrome can be delineated from seven cases. The three patients described by Gamstorp and Wohlfart (1959) show many points of similarity but distal muscular wasting was a prominent feature. In the absence of EMG it is difficult to be certain of the classification of those cases of myotonia acquisita with myokymia reviewed by Behrend (1949), but almost certainly the condition is related.

The clinical distinction of this unusual form of muscular rigidity from myotonia is difficult and most reported cases have been initially diagnosed as myotonia acquisita. The delayed relaxation is indeed indistinguishable but the characteristic feature is the continuous contraction of almost the entire skeletal musculature. Wilson (1940) remarked how infrequently the 'tonus' of the muscles in myotonia congenita at rest had been described and stated that in his experience they were not hypertonic but normally elastic to the touch. This is in contrast to the hard, almost wooden rigidity in this form of 'pseudomyotonia'. The flickering or writhing fasciculation or myokymia also appears to be a constant feature. Excessive sweating is commonly noted and is no doubt a response to the heat engendered by the constant muscular contraction. The 'stiff-man syndrome' may be distinguished by the paroxysms of severe pain and by the absence of any EMG abnormality.

The rigidity shows no response to the remedies usually effective in myotonia but responds dramatically to phenytoin or carbamazepine. In our patient the symptoms have persisted for 32 years but did not progress after the first two years. It is clear from the other published examples that such a relatively good prognosis is uncommon, although no comparable follow-up has been reported. Behrend's (1949) patient, who may have had the same condition, also improved spontaneously, but others have steadily deteriorated.

The electrophysiological findings are of some 
interest. The continuous muscle contraction is not abolished by sleep, narcosis, spinal anaesthesia (Mertens and Zschocke, 1965), or peripheral nerve block (Isaacs, 1961). It is abolished by curare (Mertens and Zschocke, 1965) or by the injection of procaine into the muscle. These results indicate that the source of the abnormal discharge lies distally in the motor unit but proximal to the motor endplate. Isaac's descriptive term 'continuous musclefibre activity' was not meant to imply that single fibres contract independently. His illustration shows discharges up to about $500 \mu \mathrm{V}$ in amplitude, but it is not possible to detect any specific pattern. Mertens and Zschocke (1965) interpreted the great variation in the amplitude of the spontaneous discharges as indicating an origin either from entire motor units, from fractions of a unit, or perhaps even from single fibres. In Sigwald and co-workers' (1966) case the amplitude of the spontaneous potentials was identical with those on voluntary activity and this was interpreted as meaning that entire motor units were discharging. In our case, however, the potentials produced by full voluntary activity are obviously of much greater amplitude than those recorded during attempted relaxation (Fig. 2e). During slight voluntary contraction this difference is not seen and the activity seems to be an augmentation of the discharges at rest (Fig. 2a) as suggested by Sigwald et al. (1966).

Our EMG evidence supports the concept that the excitation usually does not involve the entire motor unit and, therefore, that the abnormal discharges arise distal to the terminal branching of the motor fibre. The multiple repetitive discharges recorded at rest (Fig. 2b) are probably associated with the visible myokymia, although it was not possible to confirm this by direct observation. Certainly similar discharges have been recorded in benign myokymia (Denny-Brown and Foley, 1948) and in facial myokymia (Matthews, 1966). The higher voltage polyphasic prolonged discharges (Fig. $2 \mathrm{c}$ ) occurring singly more closely resemble the fasciculation potentials of neurogenic atrophy but no fibrillation potentials could be identified. Abnormal spread of excitation through the whole or part of the terminal portion of the motor unit might well result in potentials of this type, particularly as their amplitudes did not exceed approximately $800 \mu \mathrm{V}$.

No biochemical or anatomical lesion that couldo account for this syndrome has been found and we can throw no light on its cause. Some resemblances. to the hyperexcitability of tetany has been suggested (Sigwald et al., 1966), but in our patient ischaemia. did not increase the abnormal discharges but abolished them.

The beneficial effect of phenytoin and carbamaze pine was confirmed, but even after several months of treatment on the latter drug, when the patien claimed to feel normal, the electrical activity of the muscles at rest persisted.

SUMMARY

A patient is described who for 32 years has suffere from a form of muscular rigidity clinically resembling myotonia but in fact identical with the syndromes previously described as that of "continuous muscle- $N$ fibre activity'. The electrophysiological findings ape $\overrightarrow{-}$ described and the beneficial symptomatic effect of $\mathrm{f}^{-}$ certain anticonvulsants is confirmed.

\section{REFERENCES}

Behrend, R-Ch. (1949). Zur Frage der erworbenen Myotonie. D Z. Nervenheilk., 160, 22-42.

Denny-Brown, D., and Foley, J. M. (1948). Myokymia and the benign fasciculation of muscular cramps. Trans. Ass. Ame Phycns., 61, 88-96.

Gamstorp, I., and Wohlfart, G. (1959). A syndrome characterised bye myokymia, myotonia, muscular wasting and increased perspira tion. Acta psychiat. scand., 34, 181-194.

Isaacs, H. (1961). A syndrome of continuous muscle-fibre activity J. Neurol. Neurosurg. Psychiat., 24, 319-325.

Matthews, W. B. (1966). Facial myokymia. J. Neurol. Neurosurg Pyschiat., 29, 35-39.

Mertens, H-G., and Zschocke, S. (1965). Neuromyotonie. Klin. Wschr. §ิ 43, $917-925$.

Sigwald, J., Raverdy, Ph., Fardeau, M., Gremy, F., Mace de Lepinay, A., Bouttier, D., and Danic, Madame (1966). Pseudomyotonie. Forme particulière d'hypertonie musculaire a prédominance distale. Rev. neurol., 115, 1003-1014.

Wilson, S. A. K. (1940). Neurology, p. 1048. Arnold, London. 